\title{
PDGF-metronidazole-encapsulated nanofibrous functional layers on collagen membrane promote alveolar ridge regeneration
}

This article was published in the following Dove Press journal:

International Journal of Nanomedicine

2 August 2017

Number of times this article has been viewed

\author{
Ming-Hua $\mathrm{Ho}^{\prime}$ \\ Hao-Chieh Chang 2,3 \\ Yu-Chia Chang ${ }^{3}$ \\ Jeiannete Claudia' \\ Tzu-Chiao Lin ${ }^{2}$ \\ Po-Chun Chang 2,3 \\ 'Department of Chemical Engineering, \\ College of Engineering, National \\ Taiwan University of Science and \\ Technology, Taipei, Taiwan; ${ }^{2} \mathrm{Graduate}$ \\ Institute of Clinical Dentistry, \\ School of Dentistry, National Taiwan \\ University, Taipei, Taiwan; ${ }^{3}$ Department \\ of Dentistry, National Taiwan \\ University Hospital, Taipei, Taiwan
}

Correspondence: Po-Chun Chang Graduate Institute of Clinical Dentistry, School of Dentistry, National Taiwan University, I Chang-Te St, Taipei 100, Taiwan

Tel +886223123456 ext 67709

Fax +886223831346

Email changpc@ntu.edu.tw

\begin{abstract}
This study aimed to develop a functionally graded membrane (FGM) to prevent infection and promote tissue regeneration. Poly(L-lactide-co-D,L-lactide) encapsulating plateletderived growth factor (PDLLA-PDGF) or metronidazole (PDLLA-MTZ) was electrospun to form a nanofibrous layer on the inner or outer surface of a clinically available collagen membrane, respectively. The membrane was characterized for the morphology, molecule release profile, in vitro and in vivo biocompatibility, and preclinical efficiency for alveolar ridge regeneration. The PDLLA-MTZ and PDLLA-PDGF nanofibers were 800-900 nm in diameter, and the thicknesses of the functional layers were 20-30 $\mu \mathrm{m}$, with sustained molecule release over 28 days. All of the membranes tested were compatible with cell survival in vitro and showed good tissue integration with minimal fibrous capsule formation or inflammation. Cell proliferation was especially prominent on the PDLLA-PDGF layer in vivo. On the alveolar ridge, all FGMs reduced wound dehiscence compared with the control collagen membrane, and the FGM with PDLLA-PDGF promoted osteogenesis significantly. In conclusion, the FGMs with PDLLA-PDGF and PDLLA-MTZ showed high biocompatibility and facilitated wound healing compared with conventional membrane, and the FGM with PDLLA-PDGF enhanced alveolar ridge regeneration in vivo. The design represents a beneficial modification, which may be easily adapted for future clinical use.
\end{abstract}

Keywords: tissue engineering, platelet-derived growth factor, metronidazole, alveolar process, animal models

\section{Introduction}

Periodontitis is a chronic infectious disease that destroys both soft and mineralized tissues in the periodontium, leading to oral malodor, chewing insufficiency, compromised esthetics, and eventually the loss of tooth attachment. ${ }^{1}$ The prevalence rate of periodontitis is nearly $50 \%$ among adults in the USA and Taiwan. ${ }^{2,3}$ Over the last two decades, guided tissue regeneration has been regarded as the gold standard in clinical practice to reconstruct the damaged periodontium by using an occlusive membrane as a barrier to prevent the downgrowth of epithelium and to secure an environment for the ingrowth of the periodontal ligament and the alveolar bone. ${ }^{4}$

A variety of materials has been utilized to construct the barrier membrane, including expanded polytetrafluoroethylene, synthetic polymer such as polylactic acid, and naturally derived matrix such as collagen. ${ }^{5,6}$ Collagen-based membrane has been a popular choice because of its excellent cell affinity, biocompatibility, and biodegradable nature. ${ }^{4}$ However, complications such as infection due to membrane contamination is inevitable, ${ }^{7}$ and the regeneration is often limited for deep, 
noncontained intrabony defects even when bone grafting materials were supplemented. ${ }^{8}$

Functionally graded materials are composite materials with the composition and configuration that are controlled and graded to achieve the desired function. ${ }^{9}$ Bottino et $\mathrm{al}^{10}$ reported the design of a functionally graded membrane (FGM) that was composed of a core layer to provide the required mechanical properties and bioactivity, an outer electrospun layer to provide antimicrobial activity, and an inner electrospun layer to mimic the bone matrix. ${ }^{10}$ Although the design of FGM is often biologically inspired, the in vivo biocompatibility as well as the clinical feasibility of FGM in periodontal or dentoalveolar regeneration have rarely been reported and should be further explored.

In the present study, we proposed to simplify the design of FGM by adhering functional layers on the two surfaces of a clinically available regenerative membrane (Figure 1A). We encapsulated metronidazole (MTZ) in the nanofibers on the outer surface to reduce the chance of bacterial infection. MTZ is an antibiotic specific to the Gram-negative anaerobic bacteria $^{11}$ and is widely used to support dentoalveolar regeneration. ${ }^{10,12,13}$ In addition, we incorporated platelet-derived growth factor (PDGF), a potent mitogen and chemoattractant, ${ }^{14}$ in the nanofibers on the inner surface to facilitate the healing and regeneration process. We assessed the biocompatibility and applicability of this FGM design in a cell-based system and in rodent models. We hypothesized that this FGM would provide an easily adaptable modification to improve the outcome of periodontal or dentoalveolar regeneration.

\section{Materials and methods}

\section{Fabrication of the FGM}

The electrospinning setup consisted of three major components: 1) a power supply using direct current that could generate a voltage of up to $30 \mathrm{kV}$; 2) a $3 \mathrm{~mL}$ syringe attached to a metallic needle with a $0.65 \mathrm{~mm}$ inner diameter to control the flow rate of a scientific pump (model 780/00; KD Scientific, Holliston, MA, USA); and 3) a piece of aluminum foil for fiber collection. Processing variables, including the solution concentration, tip-to-collector distance, applied voltage, and the flow rate, were optimized for individual layers to obtain a bead-free fibrous structure. Poly(L-lactideco-D/L-lactide) (PDLLA; 70/30, IV: 1.6, 70LDLP200, Green Square Material Inc., Taiwan) at $6 \mathrm{wt} \%$ was dissolved in dichloromethane (DCM)/1,4-dioxane 4:6 (v:v) with the addition of $3 \% \mathrm{MTZ}, 0.3 \%(\mathrm{w} / \mathrm{w})$ PDGF, or $0.3 \%$ bovine serum albumin (BSA, in replacement of PDGF for the property assessment of functional layer), and stirred continuously for 24 hours. The dose of MTZ has been shown to inhibit Gram-negative periodontopathogens in vitro, ${ }^{15}$ and the dose of PDGF has been shown to promote dentoalveolar regeneration in vivo. ${ }^{16}$ The tip-to-collector distance was $10 \mathrm{~cm}$,
A

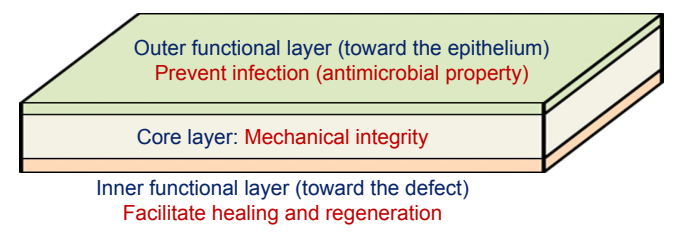

D

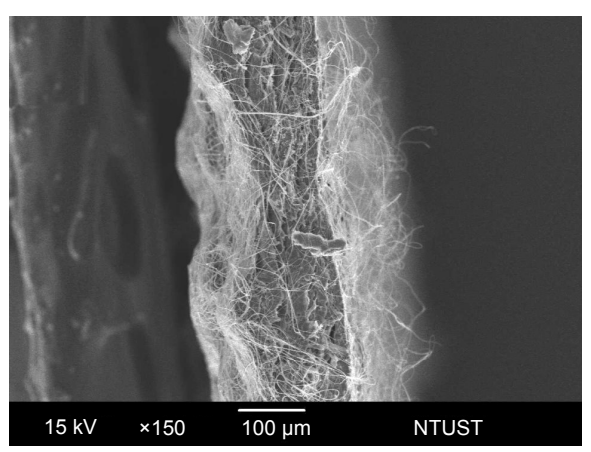

B

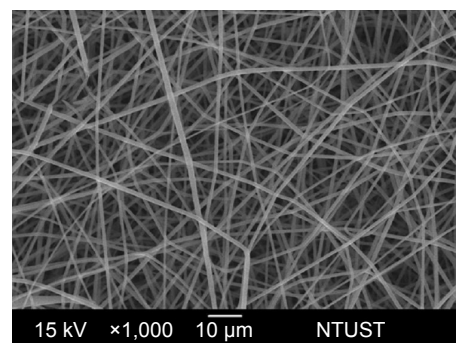

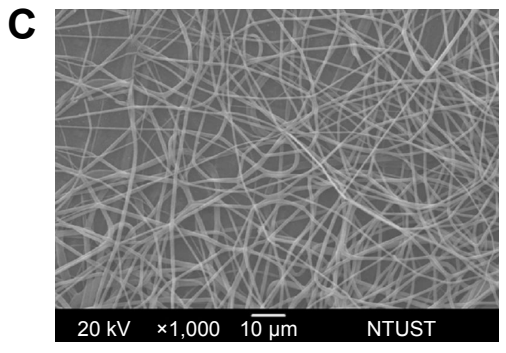

C $20 \mathrm{kV} \times 1,000 \quad \overline{10 \mu \mathrm{m}} \quad$ NTUST

\section{E}

In vitro release

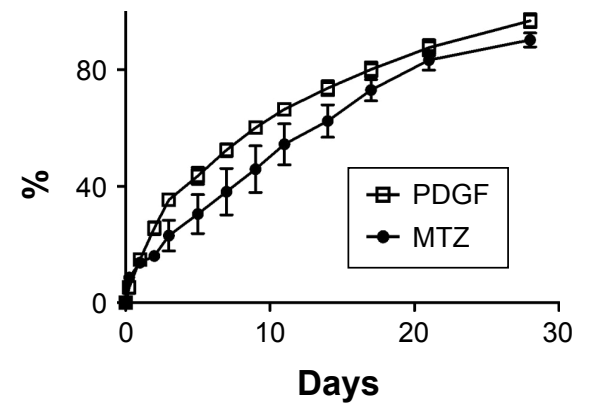

Figure I The design and characterization of the FGM.

Notes: (A) The schematic diagram of the FGM. (B) The SEM image of the PDLLA-BSA functional layer, surface scan. Magnification: I,000×. (C) The SEM image of the PDLLAMTZ functional layer, surface scan. Magnification: I,000x. (D) The SEM image of the membrane with PDLLA-BSA (on the left surface) and PDLLA-MTZ (on the right surface) functional layers, cross-sectional scan. Magnification: I50X. (E) The in vitro release profile of PDGF and MTZ from the respective nanofibrous layer.

Abbreviations: BSA, bovine serum albumin; FGM, functionally graded membrane; MTZ, metronidazole; PDGF, platelet-derived growth factor; PDLLA, poly(L-lactide-coD,L-lactide); SEM, scanning electron microscopy. 
the applied voltage was $18 \mathrm{kV}$, and the flow rate of PDLLA solution was $0.15 \mathrm{~mL} /$ hour.

The core layer of the FGM is the commercially available periodontal membrane (BioMend ${ }^{\circledR}$ Extend $^{\circledR}$, Zimmer Biomet Inc., Warsaw, IN, USA), which is composed of bovine type I collagen with the elastic modulus value of $20 \mathrm{MPa}$. The nanofibers encapsulating MTZ, PDGF, or BSA were sequentially electrospun and deposited on the surfaces of the core layer under the optimized condition described above.

\section{In vitro characterization of FGM}

For the microstructural imaging of the FGM, BSA was used in replacement of PDGF because both molecules exhibit similar affinity for an electrospun poly( $\alpha$-hydroxy ester)based scaffold. ${ }^{17}$ Individual electrospun layers and a crosssection slice of FGM were characterized by scanning electron microscopy (SEM) (JSM-6390LV, JEOL Ltd., Tokyo, Japan). The specimens were mounted on an aluminum stub, sputter coated with gold-palladium, and imaged at $15 \mathrm{keV}$. The average fiber diameter was determined by measuring the diameter of 150 fibers from each layer of three specimens at the same magnification using Image-Pro Plus software (Media Cybernetics Co., Bethesda, MD, USA).

For the analysis of the encapsulation efficiency (EE), $1 \mathrm{mg}$ of nanofibers was first dissolved in $1 \mathrm{~mL}$ of DCM, and $2 \mathrm{~mL}$ of phosphate-buffered saline (PBS, $\mathrm{pH} 7.4$ ) was subsequently added. The mixture was vortexed for 5 minutes for extraction and was then centrifuged at 9,000 rpm for 10 minutes to separate the organic and aqueous phases. The concentrations of MTZ and PDGF in the aqueous phase were measured using an UV detector (BioTek Instruments, Inc., Taiwan) at the wavelengths of 280 and $340 \mathrm{~nm}$, respectively.

The in vitro release of MTZ and PDGF was monitored over 28 days, and the cumulative release curve was plotted. Briefly, $1 \mathrm{mg}$ of nanofibers was added to a tube containing $2 \mathrm{~mL}$ of PBS, and the resultant mixture was placed in an orbital shaker bath (GFL1092, Burgwedel, Germany) at $37^{\circ} \mathrm{C}$ with constant shaking (120 rpm). The media were removed and replaced with $2 \mathrm{~mL}$ of fresh PBS at 6 hours, 24 hours, and at $2,4,7,11,14,17,21$, and 28 days. The harvested media were centrifuged at 9,000 rpm for 10 minutes, and the concentrations of MTZ and PDGF in the supernatants were measured as described above. The experiments were independently performed three times with triplicate measurements each.

\section{In vitro biocompatibility}

The in vitro biocompatibility was assessed by the metabolic activity and viability of mesenchymal stem cells (MSCs) cultured with the FGM. MSC cell line was indirectly from
Professor Junya Toguchida in Kyoto University (Kyoto, Japan), and the establishment and characterization of the cell line had been described previously. ${ }^{18}$ MSCs were cultured in Dulbecco's Modified Eagle's Medium (Sigma-Aldrich, St Louis, MO, USA) supplemented with $10 \%$ heat-inactivated fetal bovine serum (ThermoFisher Scientific Co., Waltham, MA, USA) and 1\% antibiotics (penicillin-streptomycin, ThermoFisher Scientific Co., Waltham, MA, USA). The medium was changed 2-3 times a week and the cells were maintained at $37^{\circ} \mathrm{C}$ in a humidified atmosphere of $5 \% \mathrm{CO}_{2}$ and $95 \%$ air. The nanofibers of PDLLA alone, PDLLA-MTZ, or PDLLA-PDGF were electrospun and directly deposited into the bottom of 24-well dishes. All specimens were sterilized by UV radiation for 24 hours, followed by 3 washes with PBS, and incubated with the cell culture media for 24 hours for prewetting. Cells were seeded at a density of 100,000 cells/well and cultured for 1 and 4 days before the assessment. The viability of the cells was assessed by 4,6-diamidino-2phenylindole (DAPI) assay (Sigma-Aldrich, St Louis, MO, USA) for vital cell nucleus, and by rhodamine-conjugated phalloidin (Santa Cruz Biotech Inc., Dallas, TX, USA) to label filament-actin (F-actin) for cell morphology. The metabolic activity of the cells was assessed by the Alamar Blue assay (ThermoFisher Scientific Co., Waltham, MA, USA) following the instruction of the manufacturers. Cells seeded onto uncoated wells served as the control, and all experiments were performed in triplicate.

\section{In vivo model for biocompatibility}

All procedures performed on the animals were approved by the Institutional Animal Care and Use Committee of the National Taiwan University (NTU) (Protocol No 20130087 and 20130445) following the Taiwan Animal Protection Law. Preclinical biocompatibility was evaluated by implanting FGM into the subcutaneous pouch of the mice. In brief, 12 male C57BL/6 mice (4 weeks old, weight 20-25 g) were anesthetized by intraperitoneal injection of $30 \mathrm{mg} / \mathrm{kg}$ tiletamine-zolazepam (Zoletil 50, Virbac, Cedex, France) and $0.5 \mathrm{mg} / \mathrm{kg}$ xylazine (Rompun 20, Bayer Animal Health, Monheim, Germany). Following a superficial 1-cm incision on the back, four separated subcutaneous pouches were made by blunt dissection using mosquito forceps. Four types of membranes that were $3 \times 4 \mathrm{~mm}$ in size were randomly assigned to the pouches, including the collagenous core layer only (Group CL), the core layer with the PDLLA-MTZ layer (Group MT), the core layer with the PDLLA-PDGF layer (Group PD), and the core layer with PDLLA-MTZ and PDLLA-PDGF layers (Group MP). The wound was then closed by surgical clips. The animals were euthanized by 
$\mathrm{CO}_{2}$ on days 4 and 14 , and tissue surrounding the implanted membrane was harvested for histological assessment $(n=6 /$ group/time point).

\section{In vivo model for therapeutic efficiency}

The preclinical therapeutic efficiency was evaluated by a dentoalveolar defect model in rats as previously described. ${ }^{16}$ All surgical procedures were performed under generalized anesthesia with $30 \mathrm{mg} / \mathrm{kg}$ zolazepam-tiletamine and $0.5 \mathrm{mg} / \mathrm{kg}$ xylazine. In brief, bilateral maxillary first molars (M1s) were surgically extracted from 15 male SpragueDawley rats (4 weeks old, weight 75-90 g). After 4 weeks of socket healing, an uniform osteotomy was created on each postextraction ridge of $\mathrm{M} 1$ using a customized low-speed drill at 3,000 rpm with copious normal saline irrigation. The bur was $2.6 \mathrm{~mm}$ in diameter of the cutting surface with a vertical stop at $1 \mathrm{~mm}$ in depth and $0.6 \mathrm{~mm}$ noncutting collar to ensure the consistent distance to M2. The osteotomy sites were randomly assigned to the five groups of membrane ( $\mathrm{n}=6$ /group), including no membrane control (Group CT), the membrane with the core layer only (Group CL), the membrane with the PDLLA-MTZ layer (Group MT), the membrane with the PDLLA-PDGF layer (Group PD), and the membrane with both PDLLA-MTZ and PDLLA-PDGF layers (Group MP). The test membrane was applied to cover the osteotomy site, and the wound was closed with a cyanoacrylate gel (Histoacryl ${ }^{\circledR}$; TissueSeal LLC, Ann Arbor, MI, USA). The animals were under antibiotic coverage $(268 \mathrm{mg} / \mathrm{mL}$ ampicillin in drinking water) for 7 days and euthanized on day 28.

\section{Wound dehiscence assessment}

The wound dehiscence was assessed on day 7 by a customized index based on the mesiodistal length of wound bed exposure. The mesiodistal length of M2 was defined as $100 \%$. Exposed wound bed $\geqq$ the mesiodistal length of M2 was recorded as $100 \%$, and wound bed that was completely closed was recorded as $0 \%$.

\section{Micro-computed tomography assessment}

Micro-computed tomograph (micro-CT) images were taken on days 14 and 28 by a SkyScan 1176 scanner (Bruker Corp., Kontich, Belgium), and the images were reconstructed to achieve an effective voxel size of $18 \mu \mathrm{m}$. The whole defect area was selected as the region of interest (ROI), and the threshold distinguishing the mineralized and nonmineralized tissues, the bone volume fraction (BV/TV), the trabecular thickness (Tb.Th), and the trabecular number (Tb.N) were computed for each ROI using the built-in CT-Analyser software (Bruker Corp., Kontich, Belgium).

\section{Histological assessment}

All specimens were fixed in 10\% formaldehyde for 3 days, embedded in paraffin, cut into $5 \mu \mathrm{m}$ sections, and stained with hematoxylin and eosin. For assessing biocompatibility, the membranes with attached tissues were cross-sectioned, and the thickness of fibrous encapsulation was averaged from 10 randomly selected interfacial areas on the same side of membrane for each specimen. For assessing the therapeutic efficiency, the maxillae were decalcified with $12.5 \%$ ethylenediaminetetraacetic acid and sectioned sagittally across the middle of the osteotomy sites, and the defect fill was defined as the percentage of defect area occupied with mineralized tissue, including the spaces of bone marrow and osteocyte lacunae. One additional slide in each specimen of biocompatibility assessment was stained immunohistochemically using the Cell and Tissue Staining kit (R\&D systems, Minneapolis, $\mathrm{MN}, \mathrm{USA}$ ) with a polyclonal antiproliferating cell nuclear antigen antibody (anti-PCNA, dilution 1:250; Abcam PLC., Cambridge, UK). All images were acquired using a digital image acquisition system (AxioCam ERc 5s, Carl Zeiss Microscopy GmbH, Munich, Germany).

\section{Statistical analysis}

The sample size was determined by power analysis based on a previous study. ${ }^{16}$ One-way analysis of variance followed by Tukey's post hoc test was used to compare the difference in cell behavior in vitro and the preclinical effects in vivo at each time point. An unpaired $t$-test was used for comparing the thicknesses of fibrous encapsulation between groups in vivo. All data were presented as mean $\pm \mathrm{SD}$. A $p$-value $\leq 0.05$ was considered statistically significant.

\section{Results}

\section{The design and characterization of the FGM}

The designed FGM was composed of a core layer that provided mechanical support and basic bioactivity, and two functional surface layers of composite fibers to create a locally favorable environment for tissue regeneration (Figure 1A). Figure 1B and C are representative SEM micrographs of the outer functional layer PDLLA-MTZ and the inner functional layer PDLLA-BSA, respectively. Both layers exhibited interconnected structures formed by randomly oriented nanofibers. The mean diameter of the nanofibers was $799.47 \pm 80.47 \mathrm{~nm}$ for the PDLLA-MTZ 
layer and $853.83 \pm 92.92 \mathrm{~nm}$ for the PDLLA-BSA layer. The EE was $82.19 \% \pm 15.13 \%$ for MTZ and $75.46 \% \pm 23.14 \%$ for PDGF for the respective layer. The overall FGM structure is shown in Figure 1D. The inner and outer functional layers were 20-30 $\mu \mathrm{m}$ thick each, and both were adhered onto the collagen core. Both PDGF and MTZ showed sustainedrelease profiles from the nanofibrous layers over a period of 28 days with insignificant initial burst release in the first 24 hours (Figure 1E; Table S1).

\section{The in vitro biocompatibility of the FGM}

The metabolic activity and vital cell density of MSCs were similar in the control and PDLLA-alone groups on both day 1 and day 4 (Figure 2A and B). The metabolic activity was significantly enhanced in the PDLLA-PDGF group relative to the control $(P<0.05)$, PDLLA alone $(P<0.05)$, and PDLLA-MTZ $(P<0.01)$ groups on day 1 (Figure $2 A)$. An insignificant reduction in vital cell density relative to other groups was noted for the PDLLA-MTZ group on

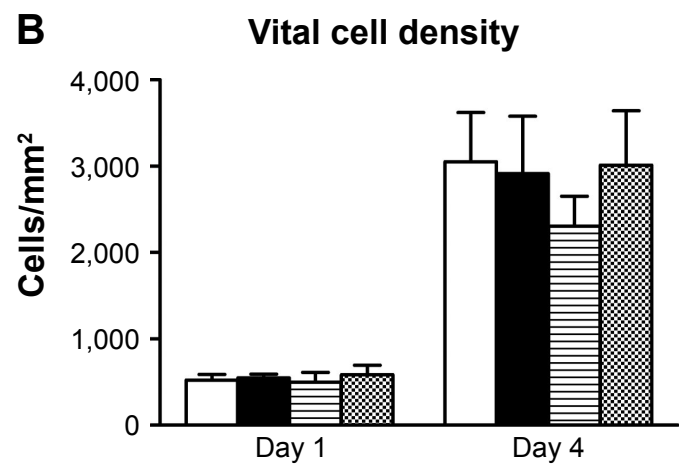

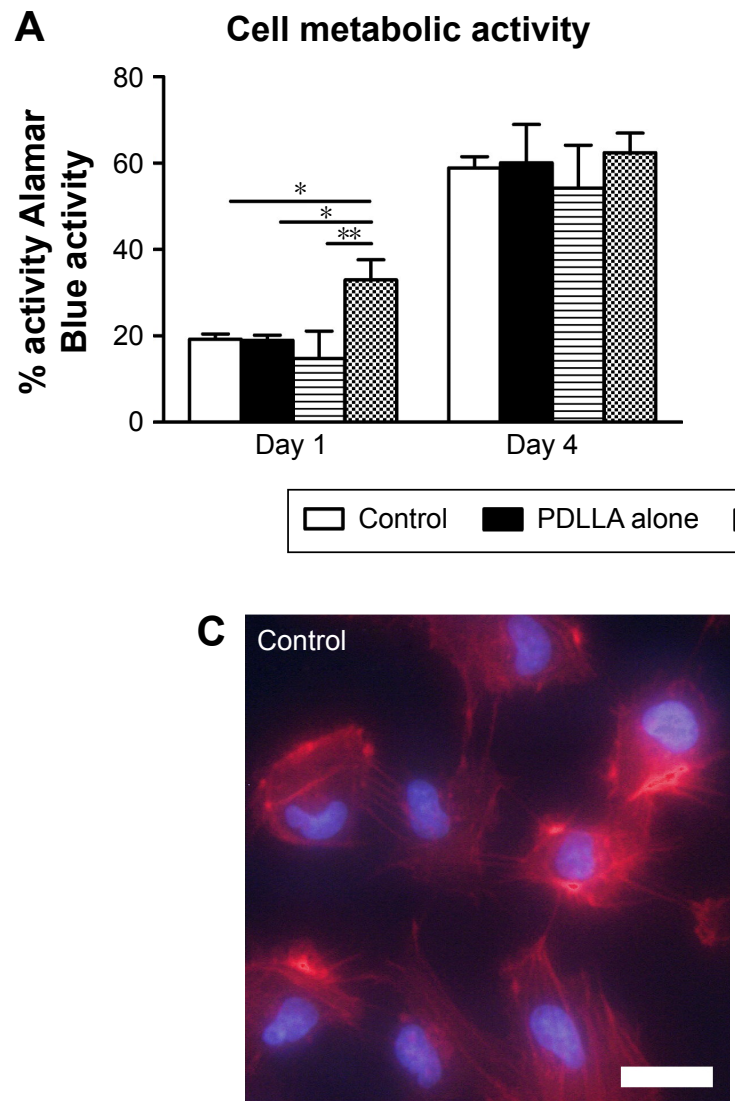
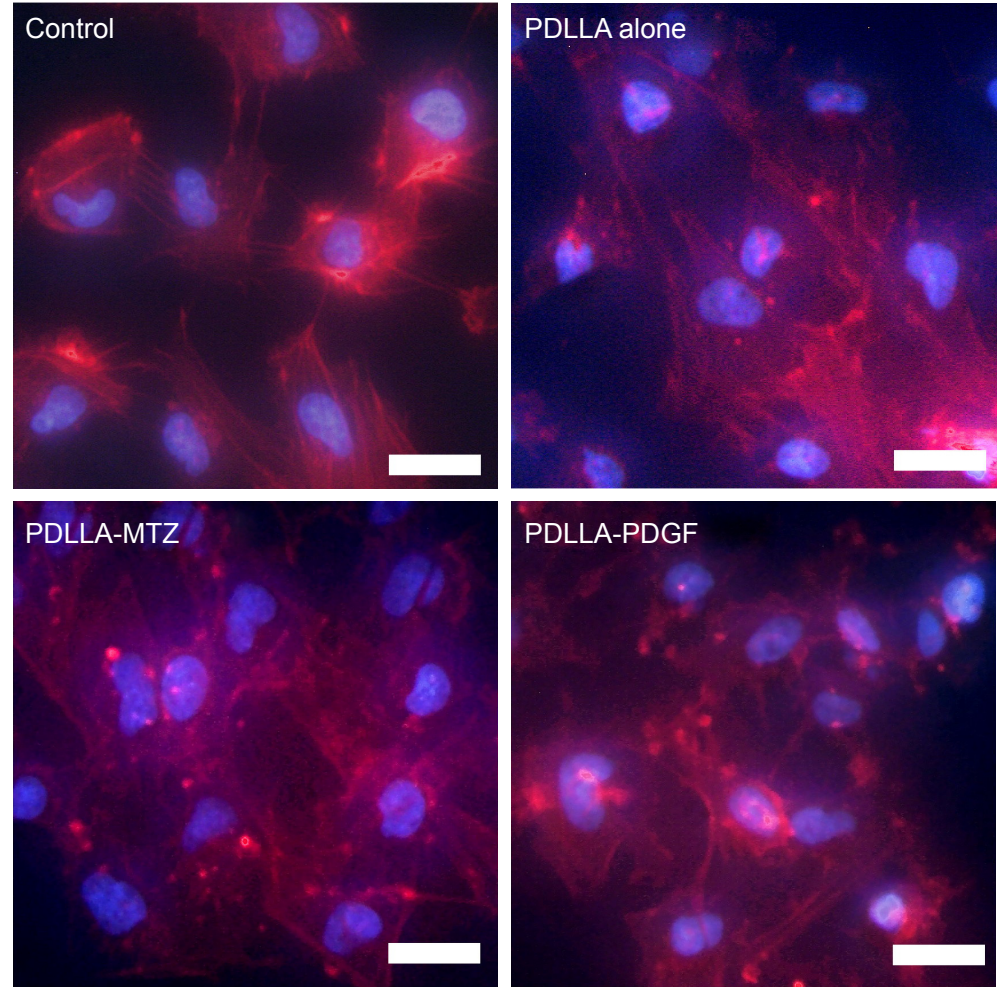

Figure $\mathbf{2}$ The in vitro assessments of FGM biocompatibility.

Notes: (A) Cell metabolic activity measured by the Alamar Blue assay. (B) Vital cell density determined by a DAPI assay. (C) Immunofluorescence images of MSCs seeded on the control and PDLLA nanofiber-coated cell culture dishes after I day. F-actin was labeled by rhodamine-conjugated phalloidin (red), and nuclei was labeled by DAPI (blue). Magnification: 400x. Scale bar: $50 \mu \mathrm{m}(* P<0.05$, **P $<0.01)$.

Abbreviations: DAPI, 4,6-diamidino-2-phenylindole; FGM, functionally graded membrane; MSCs, mesenchymal stem cells; MTZ, metronidazole; PDGF, platelet-derived growth factor; PDLLA, poly(L-lactide-co-D,L-lactide). 

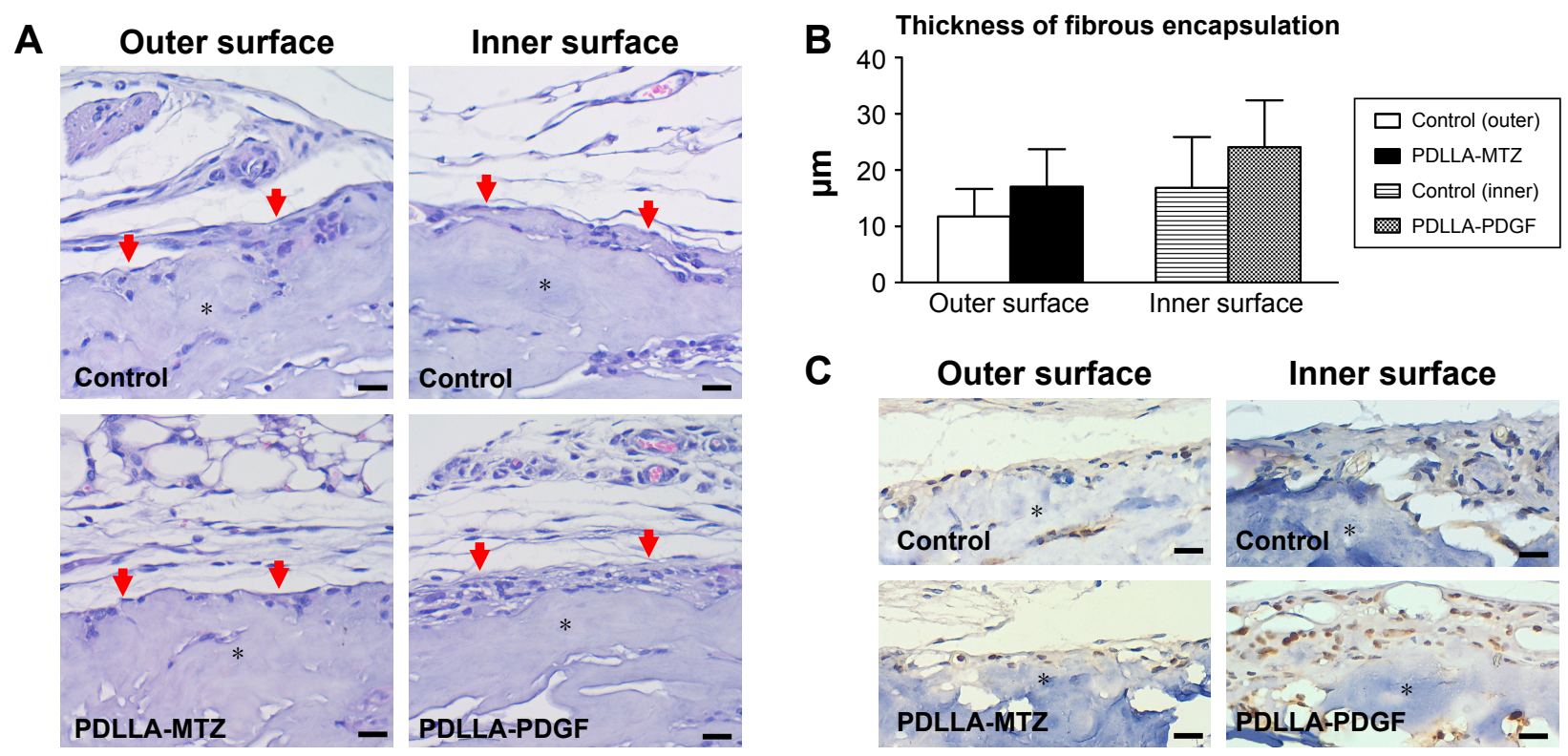

Figure 3 The preclinical assessments of FGM biocompatibility in vivo.

Notes: (A) Histology from the interface of the membrane and the surrounding subcutaneous tissue after 2 weeks of implantation. Asterisks indicate the matrix of the membrane, and red arrows indicate the fibrous encapsulation on the surface of the membrane. Magnification: 100×. Scale bar: $20 \mu \mathrm{m}$. (B) Quantification of the thickness of fibrous encapsulation. (C) Immunohistochemistry showing PCNA-positive cells (cells with brown nucleus staining) on the membrane surfaces. Asterisks indicate the matrix of the collagen core of the membrane. Magnification: I00x. Scale bar: $20 \mu \mathrm{m}$.

Abbreviations: FGM, functionally graded membrane; MTZ, metronidazole; PCNA, proliferating cell nuclear antigen; PDGF, platelet-derived growth factor; PDLLA, poly(L-lactide-co-D,L-lactide).

day 4 (Figure 2A and B). The most obvious morphological difference was observed 1 day after the cells were seeded. Compared with the control group, the three groups of cells cultured on PDLLA-coated dishes were more aggregated with anisotropically elongated F-actin (Figure 2C). There were no obvious differences with regard to the distribution of MSCs and the pattern of F-actin between the PDLLA alone, PDLLA-MTZ, and PDLLA-PDGF groups (Figure 2C). Cells were confluent with tightened F-actin filaments surrounding the nuclei in all groups at and beyond day 4 (data not shown).

\section{The preclinical biocompatibility of the FGM}

All animals that received subcutaneous implantation of membranes showed uneventful healing of the incision wound, with no visible swelling or abscess formation at any site of membrane placement. On day 4, the membranes were still intact, with scanty tissue attachment and minimal infiltration of inflammatory cells in all groups (Figure S1). On day 14, cells have attached to the membranes and produced extracellular matrix with minimal fibrous encapsulation or inflammatory infiltrate on both the inner and outer surfaces of the control membranes (upper panel of Figure 3A). Similar histological findings were noted on the surfaces of PDLLA-MTZ or PDLLA-PDGF FGMs (lower panel of Figure 3A), and the cellularity was increased on the PDLLA-PDGF layer. Fibrous encapsulation was apparently thicker on PDLLA-MTZ or PDLLA-PDGF functional layers compared with the control surfaces (Figure 3B). Furthermore, more PNCA-expressing cells were detected on the PDLLAPDGF layer suggesting enhanced proliferative potential, whereas few PCNA-positive cells were noted on the control or PDLLA-MTZ layers (Figure 3C).

\section{The preclinical efficiency of FGM}

Wound stability is the key element of dentoalveolar regeneration. In the present study, wound dehiscence was frequently seen in the first week after surgery (Figure 4) and was often prominent at the site of membrane placement. With the addition of the functional layers, the extent of wound dehiscence was reduced in both the PDLLA-MTZ (MT) and PDLLAPDGF (PD) groups compared with the core membrane control (CL). The combination of MTZ and PDGF further reduced wound dehiscence in the Group MP (Figure 4).

The micro-CT images showed that osteogenesis was more prominent at the sites treated with FGM, especially in Groups PD and MP on day 28 (Figure 5A). The quantitative measurements revealed that Groups MT and MP had insignificance increases in BV/TV and Tb.Th compared with Groups CT and CL (Figure 5B and C). Group PD showed significantly higher BV/TV than that of Groups CT 


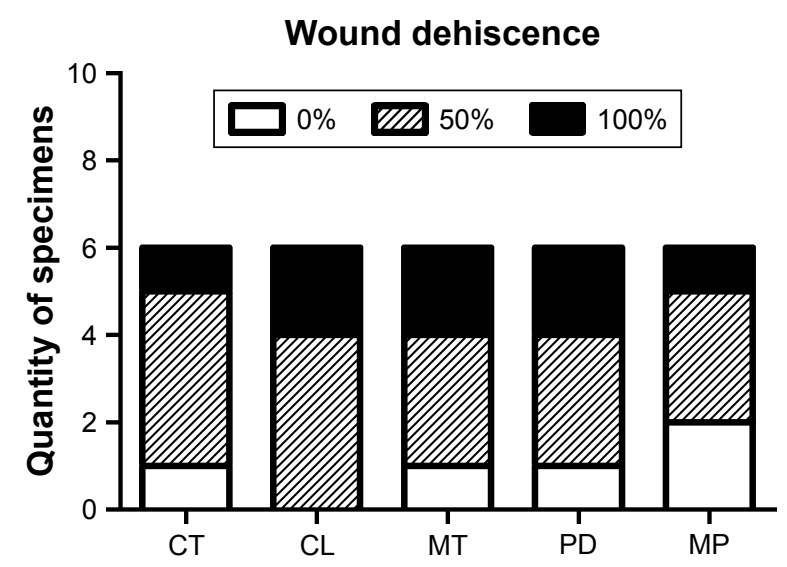

Figure 4 The extent of wound dehiscence I week after FGM application on the alveolar ridge.

Note: The results are presented as percentages of the mesiodistal length of the opened wound, with $0 \%$ indicating complete wound closure and $100 \%$ indicating completely open wound.

Abbreviations: FGM, functionally graded membrane; CT, no membrane control; $\mathrm{CL}$, membrane with the core layer only; MT, membrane with PDLLA-MTZ layer; PD, membrane with the PDLLA-PDGF layer; MP, membrane with both PDLLA-MTZ and PDLLA-PDGF layers.
A
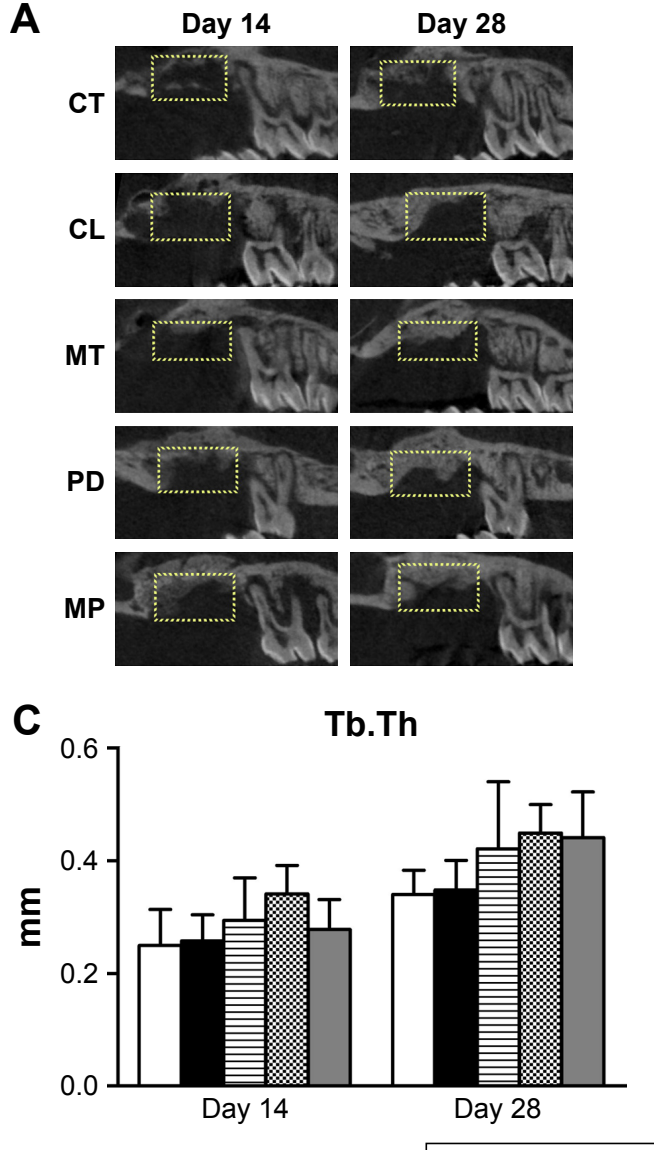

Day 28
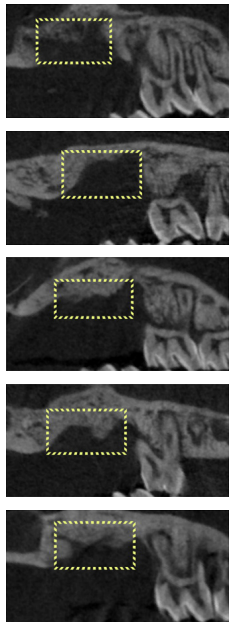

(on day $14, P<0.05$ ) and CL (on days 14 and $28, P<0.05$ for both) (Figure 5B). There was no significant difference in Tb.N among all groups on both days 14 and 28 (Figure 5D). The histological sections showed that, in Groups CT and CL, the osseous defects were mostly occupied with inflammatory cells and primitive collagen fibril network, and fragments of the residual membrane were noted in most specimens of Group CL (Figure 6A). In the defects treated with FGM, inflammation was generally subsided and the sites were filled by organized collagen matrix. In Groups PD and MP, fragments of the residual membrane were rarely seen, and osteogenesis at the peripheries of the defect was often evident. Although foreign-body giant cells were still noted surrounding the residual membrane, the membranes were integrated with the surrounding tissues in most specimens (as shown in Figure 6B). The results of histomorphometric measurement demonstrated that the defect fill was significantly greater

B
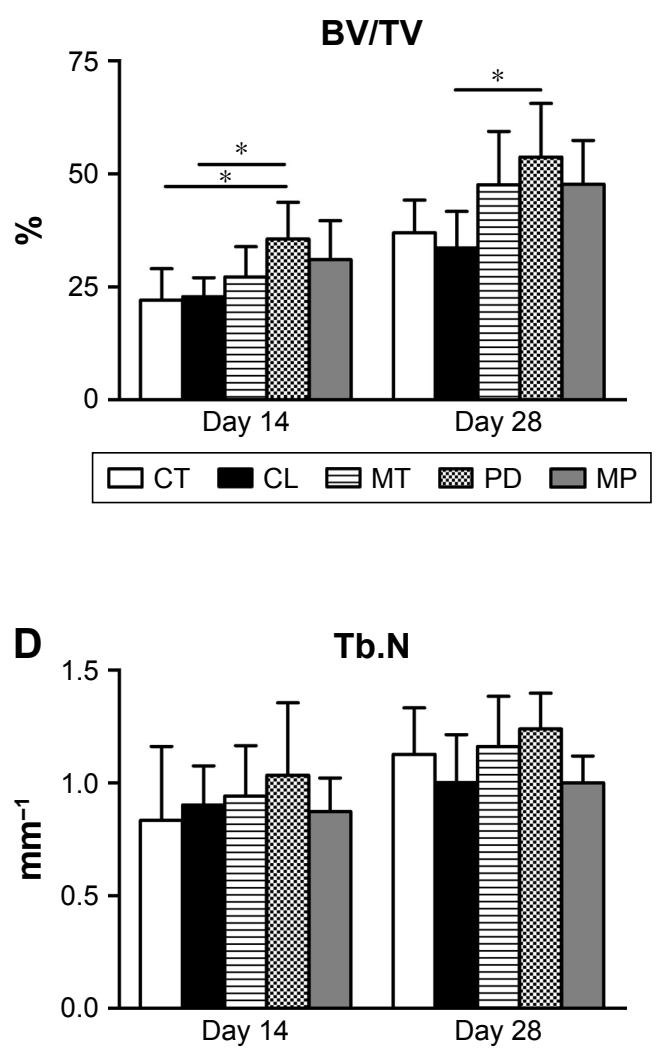

$\square \mathrm{CT} \square \mathrm{CL} \boxminus \mathrm{MT} \ggg \mathrm{PD} \square \mathrm{MP}$

Figure 5 The micro-CT assessments of the FGM on the alveolar ridge on days 14 and 28.

Notes: (A) The representative sagittal sections from each group on days 14 and 28. Dash boxes indicate the osseous defects. (B) The bone volume fraction, defined as the ratio of bone volume to the entire volume of defect (BV/TV). (C) The mean trabecular thickness (Tb.Th). (D) The mean trabecular number (Tb.N) per square millimeter. $* P<0.05$.

Abbreviations: BV/TV, bone volume fraction; FGM, functionally graded membrane; Tb.N, trabecular number; Tb.Th, trabecular thickness; CT, no membrane control; $\mathrm{CL}$, membrane with the core layer only; MT, membrane with PDLLA-MTZ layer; PD, membrane with the PDLLA-PDGF layer; MP, membrane with both PDLLA-MTZ and PDLLA-PDGF layers. 
A
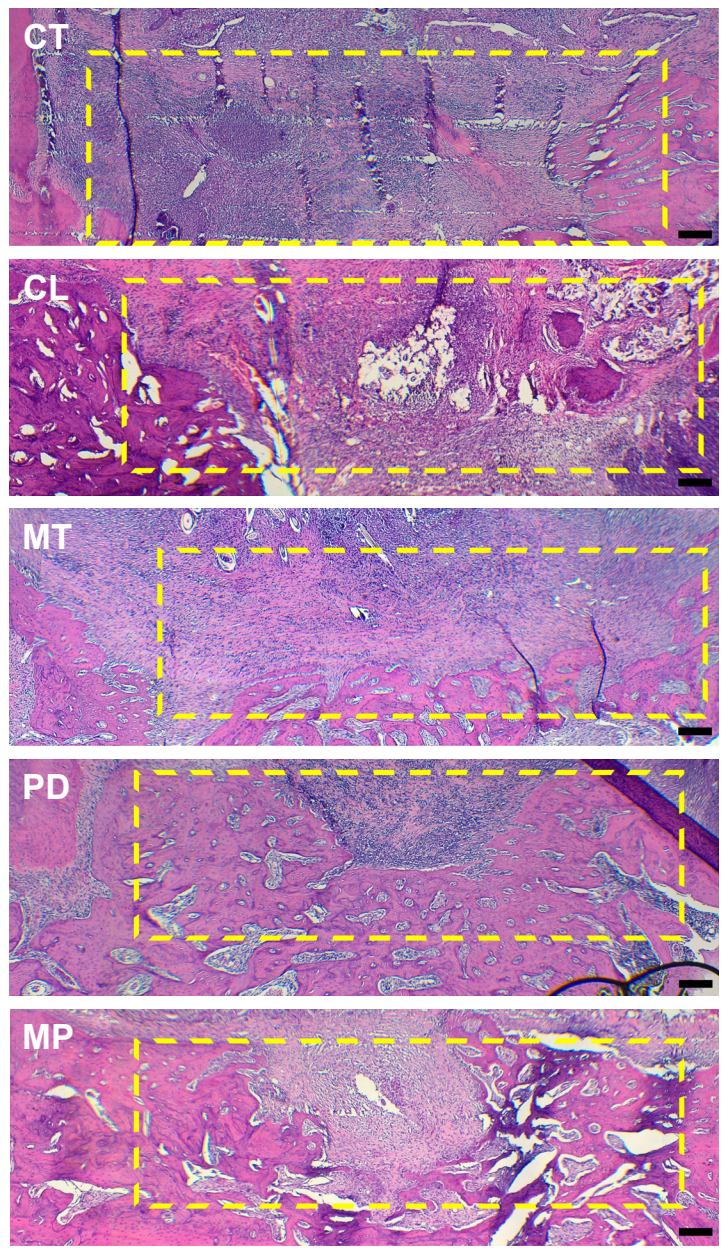

B
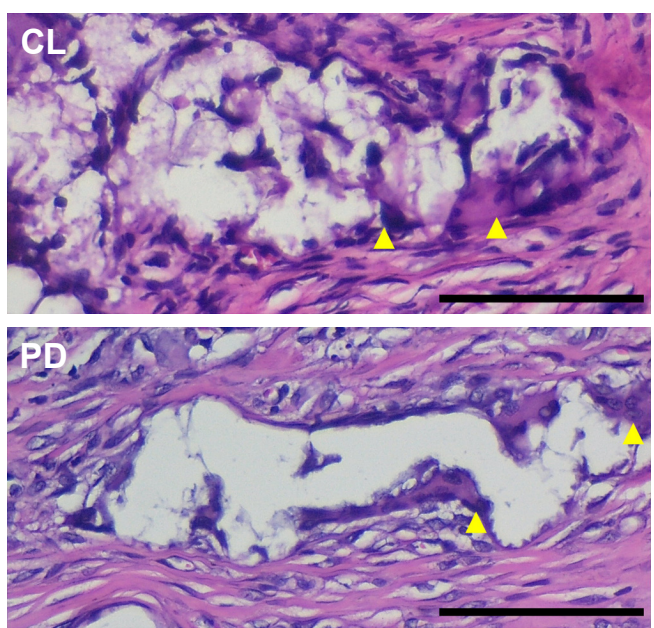

C

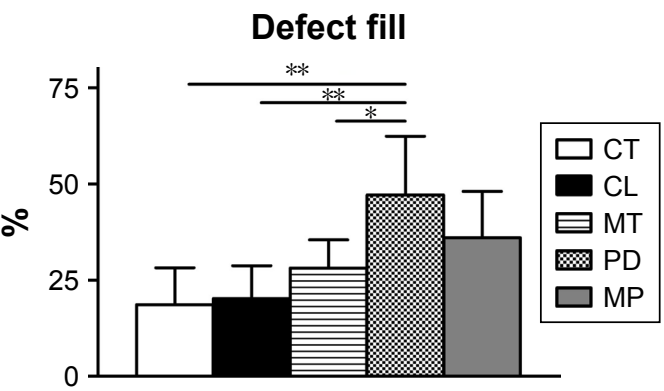

Figure 6 The histologic assessments of the FGM on the alveolar ridge on day 28.

Notes: (A) The representative sagittal section of each group. Dash boxes indicate the osseous defects. Magnification: $40 \times$. Scale bar: $200 \mu \mathrm{m}$. (B) Tissue surrounding the residual membrane in Groups CL (upper panel) and PD (lower panel). Arrowheads indicate foreign-body giant cells. Magnification: 400×. Scale bar: I00 $\mu$ m. (C) Histomorphometric assessment for the defect fill, which was defined as the percentage of defect area occupied with mineralized tissue $(* P<0.05, * * P<0.01)$.

Abbreviations: FGM, functionally graded membrane; CT, no membrane control; CL, membrane with the core layer only; MT, membrane with PDLLA-MTZ layer; PD, membrane with the PDLLA-PDGF layer; MP, membrane with both PDLLA-MTZ and PDLLA-PDGF layers.

in Group PD compared with Groups CT $(P<0.01)$, CL $(P<0.01)$, or MT $(P<0.05)$ (Figure 6C).

\section{Discussion}

In this study, we designed an FGM, which had a core layer to provide the required mechanical properties and basic bioactivity, and two functional surface layers of composite nanofibers to facilitate tissue integration (Figure 1A). ${ }^{10}$ We chose a commercially available periodontal membrane composed of bovine type I collagen as the core layer because of its high biocompatibility and superior effect in promoting cell attachment, proliferation, and differentiation, comparing with other periodontal membranes. ${ }^{19}$ The functional surface layers were fabricated with PDLLA for the degradation property and biocompatibility. ${ }^{17,20}$ The functional layers were created by electrospinning technology, which is a simple and well-documented method to fabricate micro- and nanoscale fibers without compromising the chemical characteristics of the polymers or the encapsulated drugs. ${ }^{11,21,22}$ The higher surface area-to-volume ratio of electrospun fibers has been shown to adsorb vitronectin and fibronectin to facilitate cell adhesion and proliferation. ${ }^{15,22}$ As expected, we found that cells were well attached to the PDLLA nanofibrous layer and exhibited equivalent viability as those on the control surface (Figure 2). To further promote healing and regeneration, MTZ was encapsulated in the outer functional layer at a dosage effective to inhibit periodontopathogen, whereas PDGF was encapsulated in the inner functional layer to promote mitogenesis. ${ }^{11,14-16}$ The sustained release of MTZ and PDGF (Figure 1E) inhibited of biofilm formation ${ }^{23}$ and enhanced the chemotaxis of MSCs,${ }^{24}$ suggesting that FGM may exert beneficial effects in vivo. 
Biocompatibility is one of the major prerequisites in the development of new functional biomaterials. ${ }^{25}$ In addition to the effect on cell behavior, foreign body reaction and tissue damage are also major concerns for the evaluation of preclinical biocompatibility. Foreign body reaction is often provoked by polymers (ie, PDLLA) and leads to the infiltration of macrophages and polymorphonuclear cells attempting to remove the polymers. ${ }^{26}$ If foreign body reaction persists, the material would be walled off by a fibrous capsule, which prevents it from interacting with the surrounding tissue. Moreover, the degradation of poly ( $\alpha$-hydroxy ester) releases acidic byproducts that may release free radicals and cause cell death, resulting in tissue damage. ${ }^{27}$ In our subcutaneous implantation model, inflammatory infiltration and fibrous encapsulation surrounding the FGM were minor (Figure 3A and $\mathrm{B})$. Compared with the control membrane, increased cell proliferation was noted on the PDGF-encapsulated functional layers (Figure 3C). These results confirmed the high biocompatibility of this FGM and the potential benefit of a PDGF-loaded functional layer.

We evaluated the preclinical applicability of the FGM in a rat model of large-sized dentoalveolar defects. Although this type of surgically created defect may not be clinically relevant to alveolar ridge deficiencies in humans, the standardized critical-sized defect in the same anatomical region is still informative as an initial investigation. ${ }^{16}$ In the present study, wound dehiscence was often seen after the surgery, possibly due to the thin gingiva of the rats that is more susceptible to recession, and the inability to control for plaque and the chewing behaviors of rats. ${ }^{28}$ Reduced wound dehiscence in the defects treated with all of the FGM configurations tested, as shown in Figure 4, may be associated with high cell affinity for the nanofibrous structure ${ }^{15}$ and it may also imply that effective infection control and/or enhanced chemotaxis and mitogenesis may promote wound healing. ${ }^{29,30}$ According to the micro-CT imaging and the histological assessments, both BV/TV and defect fill were significantly enhanced in Group PD relative to Groups CT and CL (Figures 5B and 6C), supporting that FGM with PDGF-loaded nanofibrous surface layer accelerated the regeneration of large dentoalveolar defects but did not significantly influence the quality of osteogenesis. Similar trend was also shown in our previous study, which showed that PDGF supplied from sustained-release microspheres can promote dentoalveolar osteogenesis. ${ }^{16}$ These studies demonstrate the benefits of incorporating exogenous growth factors in promoting the healing of large-sized dentoalveolar defects. However, complete defect resolution was not achieved in this study, regardless of the treatment groups (Figure 6). One possible explanation was the early collapse of the membrane during regeneration because of insufficient mechanical support, which could not resist the external load from the oral environment. In the future, the strength of the FGM may be improved by combining the FGM with grafting materials or further precipitating hydroxyapatite onto the FGM to maximize its effect on dentoalveolar regeneration. ${ }^{4}$

\section{Conclusion}

We created an FGM by depositing functional layers composed of electrospun PDLLA nanofibers encapsulating MTZ or PDGF on a commercially available collagen periodontal membrane. The FGM exhibited good biocompatibility, and FGM with the PDGF-encapsulated functional layer was effective in preventing wound dehiscence and accelerating the regeneration process. This study provided a feasible and economical approach to adapt an easily available periodontal membrane for an enhanced regeneration capacity. Future study is warranted to further improve the design of the functional layers and to investigate how the FGM maintains the defect space for the optimization of tissue regeneration.

\section{Acknowledgments}

The authors express their gratitude to the Material Science and Technology Center, College of Engineering, National Taiwan University of Science and Technology for providing instrumentation facilities. The authors are also thankful to the Laboratory Animal Center, College of Medicine, NTU, for the assistance in managing the micro-CT imaging and histological slides. The study was supported by research grants EX106-10313EC from the National Health Research Institutes (Taiwan), 105-2314-B-002-084 from the Ministry of Science and Technology (Taiwan), and 106-N3615 from the National Taiwan University Hospital (Taiwan).

\section{Disclosure}

The authors report no conflicts of interest in this work.

\section{References}

1. Haffajee AD, Socransky SS. Microbial etiological agents of destructive periodontal diseases. Periodontol 2000. 1994;5:78-111.

2. Albandar JM, Brunelle JA, Kingman A. Destructive periodontal disease in adults 30 years of age and older in the United States, 1988-1994. J Periodontol. 1999;70(1):13-29.

3. Lai H, Su CW, Yen AM, et al. A prediction model for periodontal disease: modelling and validation from a national survey of 4061 Taiwanese adults. J Clin Periodontol. 2015;42(5):413-421.

4. Bottino MC, Thomas V, Schmidt G, et al. Recent advances in the development of GTR/GBR membranes for periodontal regeneration-a materials perspective. Dent Mater. 2012;28(7):703-721. 
5. Corinaldesi G, Lizio G, Badiali G, Morselli-Labate AM, Marchetti C. Treatment of intrabony defects after impacted mandibular third molar removal with bioabsorbable and non-resorbable membranes. JPeriodontol. 2011;82(10):1404-1413.

6. Vouros I, Aristodimou E, Konstantinidis A. Guided tissue regeneration in intrabony periodontal defects following treatment with two bioabsorbable membranes in combination with bovine bone mineral graft. A clinical and radiographic study. J Clin Periodontol. 2004;31(10): 908-917.

7. Chen YT, Hung SL, Lin LW, Chi LY, Ling LJ. Attachment of periodontal ligament cells to chlorhexidine-loaded guided tissue regeneration membranes. J Periodontol. 2003;74(11):1652-1659.

8. Iorio-Siciliano V, Andreuccetti G, Blasi A, Matarasso M, Sculean A, Salvi GE. Clinical outcomes following regenerative therapy of noncontained intrabony defects using a deproteinized bovine bone mineral combined with either enamel matrix derivative or collagen membrane. J Periodontol. 2014;85(10):1342-1350.

9. Almasi D, Sadeghi M, Lau WJ, Roozbahani F, Iqbal N. Functionally graded polymeric materials: a brief review of current fabrication methods and introduction of a novel fabrication method. Mater Sci Eng C Mater Biol Appl. 2016;64:102-107.

10. Bottino MC, Thomas V, Janowski GM. A novel spatially designed and functionally graded electrospun membrane for periodontal regeneration. Acta Biomater. 2011;7(1):216-224.

11. Reise M, Wyrwa R, Muller U, et al. Release of metronidazole from electrospun poly(L-lactide-co-D/L-lactide) fibers for local periodontitis treatment. Dent Mater. 2012;28(2):179-188.

12. Liu Y, Zhang X, Liu Y, et al. Bi-functionalization of a calcium phosphatecoated titanium surface with slow-release simvastatin and metronidazole to provide antibacterial activities and pro-osteodifferentiation capabilities. PLoS One. 2014;9(5):e97741.

13. Kurtis B, Unsal B, Cetiner D, et al. Effect of polylactide/glycolide (PLGA) membranes loaded with metronidazole on periodontal regeneration following guided tissue regeneration in dogs. J Periodontol. 2002; 73(7):694-700.

14. Kaigler D, Avila G, Wisner-Lynch L, et al. Platelet-derived growth factor applications in periodontal and peri-implant bone regeneration. Expert Opin Biol Ther. 2011;11(3):375-385.

15. Xue J, He M, Niu Y, et al. Preparation and in vivo efficient antiinfection property of GTR/GBR implant made by metronidazole loaded electrospun polycaprolactone nanofiber membrane. Int J Pharm. 2014;475(1-2):566-577.

16. Chang PC, Chong LY, Dovban AS, et al. Sequential platelet-derived growth factor-simvastatin release promotes dentoalveolar regeneration. Tissue Eng Part A. 2014;20(1-2):356-364.

17. Chang PC, Chung MC, Lei C, Chong LY, Wang CH. Biocompatibility of PDGF-simvastatin double-walled PLGA (PDLLA) microspheres for dentoalveolar regeneration: a preliminary study. J Biomed Mater Res A. 2012;100(11):2970-2978.
18. Okamoto T, Aoyama T, Nakayama T, et al. Clonal heterogeneity in differentiation potential of immortalized human mesenchymal stem cells. Biochem Biophys Res Commun. 2002;295(2):354-361.

19. Takata T, Wang HL, Miyauchi M. Attachment, proliferation and differentiation of periodontal ligament cells on various guided tissue regeneration membranes. J Periodontal Res. 2001;36(5):322-327.

20. Ma Z, Chen F, Zhu YJ, Cui T, Liu XY. Amorphous calcium phosphate/ poly(D,L-lactic acid) composite nanofibers: electrospinning preparation and biomineralization. J Colloid Interface Sci. 2011;359(2):371-379.

21. Ho MH, Yao CJ, Liao MH, Lin PI, Liu SH, Chen RM. Chitosan nanofiber scaffold improves bone healing via stimulating trabecular bone production due to upregulation of the Runx2/osteocalcin/alkaline phosphatase signaling pathway. Int J Nanomedicine. 2015;10:5941-5954.

22. Li WJ, Cooper JA Jr, Mauck RL, Tuan RS. Fabrication and characterization of six electrospun poly(alpha-hydroxy ester)-based fibrous scaffolds for tissue engineering applications. Acta Biomater. 2006;2(4): 377-385.

23. Kim K, Luu YK, Chang C, et al. Incorporation and controlled release of a hydrophilic antibiotic using poly(lactide-co-glycolide)-based electrospun nanofibrous scaffolds. J Control Release. 2004;98(1):47-56.

24. Phipps MC, Xu Y, Bellis SL. Delivery of platelet-derived growth factor as a chemotactic factor for mesenchymal stem cells by bone-mimetic electrospun scaffolds. PLoS One. 2012;7(7):e40831.

25. Dreifke MB, Ebraheim NA, Jayasuriya AC. Investigation of potential injectable polymeric biomaterials for bone regeneration. $J$ Biomed Mater Res A. 2013;101(8):2436-2447.

26. Morais JM, Papadimitrakopoulos F, Burgess DJ. Biomaterials/tissue interactions: possible solutions to overcome foreign body response. AAPS J. 2010;12(2):188-196.

27. Cao L, Duan PG, Wang HR, et al. Degradation and osteogenic potential of a novel poly(lactic acid)/nano-sized beta-tricalcium phosphate scaffold. Int J Nanomedicine. 2012;7:5881-5888.

28. Tao CY, Lee N, Chang HC, Yang C, Yu XH, Chang PC. Evaluation of $660 \mathrm{~nm}$ LED light irradiation on the strategies for treating experimental periodontal intrabony defects. Lasers Med Sci. 2016;31(6): 1113-1121.

29. Mariano RC, Oliveira MR, Silva LC, Ferreira S, Garcia Junior IR, de Carvalho Silva A. Effect of topical application of chlorhexidine and metronidazole on the tissue repair of palatal wounds of rats: a clinical and histomorphometric study. Oral Surg Oral Med Oral Pathol Oral Radiol. 2015;119(5):505-513.

30. Travis TE, Mauskar NA, Mino MJ, et al. Commercially available topical platelet-derived growth factor as a novel agent to accelerate burn-related wound healing. J Burn Care Res. 2014;35(5):e321-e329. 


\section{Supplementary materials}

Table SI Cumulative release of the encapsulated molecules from the nanofibers

\begin{tabular}{lll}
\hline & PDLLA-MTZ (\%) & PDLLA-PDGF (\%) \\
\hline 6 hours & $8.63 \pm 1.85$ & $5.21 \pm 2.03$ \\
24 hours & $13.72 \pm 1.37$ & $14.74 \pm 1.66$ \\
2 days & $16.02 \pm 1.66$ & $25.55 \pm 2.18$ \\
3 days & $23.00 \pm 5.30$ & $35.24 \pm 1.53$ \\
5 days & $30.38 \pm 6.74$ & $43.54 \pm 2.89$ \\
7 days & $38.07 \pm 7.98$ & $52.36 \pm 2.17$ \\
9 days & $45.80 \pm 7.95$ & $60.02 \pm 1.95$ \\
11 days & $54.33 \pm 7.03$ & $66.38 \pm 1.94$ \\
14 days & $62.31 \pm 5.58$ & $73.61 \pm 2.55$ \\
17 days & $72.94 \pm 3.67$ & $80.04 \pm 2.57$ \\
21 days & $83.22 \pm 3.41$ & $87.52 \pm 2.76$ \\
28 days & $90.17 \pm 2.40$ & $96.79 \pm 2.41$ \\
\hline
\end{tabular}

Note: The data are presented as mean \pm SD.

Abbreviations: PDLLA-MTZ, poly(L-lactide-co-D,L-lactide) encapsulating metronidazole; PDLLA-PDGF, poly(L-lactide-co-D,L-lactide) encapsulating plateletderived growth factor.

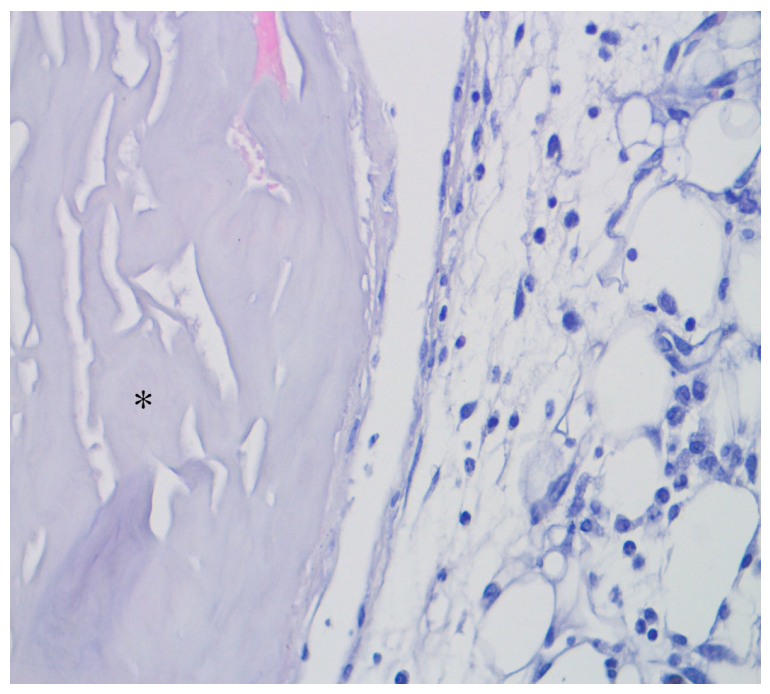

Figure SI A representative histology image showing the interface of the control membrane and the surrounding subcutaneous tissue on day 4 after implantation. Notes: Scanty tissue attachment and minimal infiltration of inflammatory cells was noted on the surface of the membrane in all groups. Asterisk indicates the residual membrane.

\section{Publish your work in this journal}

The International Journal of Nanomedicine is an international, peerreviewed journal focusing on the application of nanotechnology in diagnostics, therapeutics, and drug delivery systems throughou the biomedical field. This journal is indexed on PubMed Central, MedLine, CAS, SciSearch ${ }^{\circledR}$, Current Contents ${ }^{\circledR} /$ Clinical Medicine,

\section{Dovepress}

Journal Citation Reports/Science Edition, EMBase, Scopus and the Elsevier Bibliographic databases. The manuscript management system is completely online and includes a very quick and fair peer-review system, which is all easy to use. Visit http://www.dovepress.com/ testimonials.php to read real quotes from published authors. 